\title{
Design of Ferromagnetic Resonance Monitoring System of Electric System
}

\author{
Zhe Yu \\ Shenyang Institute of Engineering, Liaoning, Shenyang110000 \\ huner2011@foxmail.com
}

Keywords: Electric system, Ferromagnetic resonance, Monitoring system

\begin{abstract}
The paper designs a ferromagnetic resonance monitoring system of electric system, and the key problem is to select system microprocessor. The paper firstly researches and analyzes the actual research and model selection of ARM chip in the design, and expounds type selection principle of ferromagnetic resonance monitoring system, and analyzes the characteristics of LPC2214 ARM microprocessor of the system. Then, the paper proposes the requirements and design objective of the system. Lastly, the paper proposes the design scheme of the ferromagnetic resonance monitoring system.
\end{abstract}

\section{Model Selection of ARM Microprocessor}

Structure of ARM microprocessor. ARM microprocessor has 37 registers, and is divided into several groups (Bank). The registers include,

31 common registers including program counter (PC pointer), which are 32-bit registers.

6 state registers, which are used to identify working condition of CPU and running status of program and are 32 bit. And only some are used.

ARM processor has 7 different processor modes, Under each processor mode, there are a group of registers, which means that under any processor mode, the addressable registers include 15 common registers $(\mathrm{R} 0 \sim \mathrm{R} 14)$, one or two state registers and program counters.

In RISC architecture, ARM microprocessor supports two instruction sets, ARM instruction set and Thumb instruction set. The length of ARM instruction is $32 \mathrm{bit}$, and the length of Thumb instruction is 16 bit. Thumb instruction set is functional subset $\mathrm{f}$ ARM instruction set, which not only can save $30 \% \sim 40 \%$ memory space compared with equivalent ARM code, but also has the advantages of 32-bit code.

Type selection of ARM Microprocessor Application of System. ARM microprocessor has many advantages. With the development of embedded application fields at home and abroad, ARM microprocessor is widely applied. However, ARM microprocessor has more than ten kernel structures, has many manufactures of chips, and various internal functional configuration combinations, which makes developers difficult to make selection schemes. How to select a proper ARM microprocessor for ferromagneticv resonance monitoring system of electric system is an important problem in the initial stage of constructing system.

Starting from the perspective of application, the paper analyzes the selection of ARM microprocessor, as follows.

Selection of ARM microprocessor core. ARM microprocessor includes a series of kernel structures to adapt to different application fields. If the users hope to use WinCE or standard Linux to reduce software development time, it needs t select ARM720T with ARM chip of MMU

(Memory Management Unit), such as ARM720T and ARM920T with MMU function. But ARM7TDMI has no MMU and doesn't support Windows CE and standard Linux. But $\mu \mathrm{C} / \mathrm{OS}$ can be operated on ARM7TDMI hardware platform without needing operation system supported by MMU. In fact, $\mu \mathrm{C} / \mathrm{OS}$ has been successfully implanted into many microprocessor platforms without MMU, and has advantages for stability. LPC2214 for the system is an ARM microprocessor without MMU and can operate $\mu \mathrm{C} / \mathrm{OS}$ operation system, which meets the functional requirement of the system. 
Working frequency of system. Working frequency of system determines processing ability of ARM microprocessor. The typical processing velocity of ARM7 series microprocessor is $0.9 \mathrm{MIPS} / \mathrm{MHz}$, and the master clock of common ARM7 chip system is $20 \mathrm{MHz} \sim 133 \mathrm{MHz}$. The maximum clock frequency of LPC2214 is 60MHz.

Capacity of internal memory of chip. The capacity of internal memory of most microprocessors is not large, which requires the users to expand the memory. But some chips have large memory space such as the internal program memory space of AT91F40162 of ATMEL achieves $2 \mathrm{MB}$, and the users can select the type to simplify the system design. LPC2214 integrates 256KB Flash program memory, which completely meets the requirement of the system.

Selection of on-chip peripheral circuit. Except for ARM microprocessor core, nearly all ARM chips expand functional module according to different application fields and are integrated into chips, which are called on-chip peripheral circuit such as USB interface, I2S interface, LCD controller, keyboard interface, RTC, ADC and DAC, and DSP co-processor. LPC2214 has eight-road 10-bit approximate ADC, timing clock and watchdog, which meets the requirement of design.

\section{Technical Parameters of ARM LPC2214}

LPC2214 is a 16/32-bit ARM7TDMI-STMCPU microprocessor supporting real-time emulation and trace, and has 256KB embedded high-speed on-chip Flash memory. On-chip 128-bit memory interface and unique accelerating structure makes 32-bit code operate under the maximum clock frequency. 16-bit Thumb mode can be used to make the code scale reduce by $30 \%$, which makes small performance loss.

LPC2214 has small 144-pin packages, low power consumption, several 32-bit timers, 8-road 10-bit ADC and more than 9 external interruption, which makes it appropriate for industrial control, medical system, access control and POS machine.

LPC2214 can use 76 GPIO (using external memory) 112 (single-chip application). As wide-range serial communication interface is built in, it is applicable for communication gateway, protocol converter, embedded software Modern and application of other types.

\section{Function Design of Ferromagnetic Resonance Monitoring System of Electric System}

Ferromagnetic resonance monitoring system of electric system should have the following functions.

(1) Ferromagnetic resonance analysis and resonance eliminating function

The system detects PT triangular voltage value. When the power grid happens ferromagnetic resonance, the system can accurately measures and analyzes the voltages of $17 \mathrm{~Hz}, 25 \mathrm{~Hz}, 50 \mathrm{~Hz}$ and $150 \mathrm{~Hz}$, automatically triggers both sides of solid-state relay to instantly break-over PT triangle to break the condition of PT and line resonance and dynamically eliminates ferromagnetic resonance of electric system.

(2) Failure alarming function: When the system has the failure of ferromagnetic resonance, single ground connection or over voltage, it has the indication and alarm signal.

(3) Display function: It displays the operation state of system, and the starting time of ferromagnetic resonance, single ground connection or over voltage.

(4) Parameter setting function: The users can set or modify the threshold voltage, real-time clock and communication way of resonance eliminating enablement.

(5) Communication function: The system has RS-485 communication interface, perfect communication function, and the communication rate is optional.

\section{Overall Structure of Ferromagnetic Resonance Monitoring System of Electric System}

Hardware structure of system. According to the functional requirement analysis of system, the overall hardware structure of system is determined. Ferromagnetic resonance monitoring system of 
electric system can be divided into signal selection module, signal conditioning module, ferromagnetic resonance detection and analysis module, resonance eliminating module, communication module, monitoring module and external memory module.

Signal collection module converts PT triangle voltage $0 \sim 120 \mathrm{~V}$ voltage signal into $0 \sim 3 \mathrm{~V}$ voltage signal by voltage converter. And zero-cross detection is used to ensure that the sampling time is in a signal period. Sampling signal conditioning module makes appropriate conditioning and conversion on the collected signals. It not only makes the signals meet the voltage range of ARM chip inputting pin, but also provides reliable and effective signals for ferromagnetic resonance detection module. Ferromagnetic resonance detection and analysis module samples certain points on the inputted simulation signals by A/D converting unit of LPC2214. After FFT conversion, the required voltage component amplitude under the frequency of $17 \mathrm{~Hz}, 25 \mathrm{~Hz}, 50 \mathrm{~Hz}$ and $150 \mathrm{~Hz}$ required by the users is achieved. The function of resonance eliminating module is to break the condition of PT and line resonance and dynamically eliminate ferromagnetic resonance of electric system. Communication module provides the network communication of ARM and upper computer, and sends the failure information of electric system to the upper computer. Monitoring module provides the required data display and keyboard control, and makes indication alarm on failures. The external memory is designed for enhancing data processing capability of system.

Software structure of system. Most traditional embedded system designs use single-task sequence mechanism. The application is an infinite cycle, all events are executed orderly, and the events relating to time is ensured according to timer interruption. The advantage of the programming mode is direct, and the problem is worse stability and instantaneity of system. Especially when the system functions are complicated, and it has strict requirement on real time, the disadvantages of of single-task mechanism are evident. So in the design of the ferromagnetic resonance monitoring system of electric system, $\mu \mathrm{C} / \mathrm{OS}$ - II real-time operation system is applied to program design of LPC2214. The functions which are implemented by the system is divided into several core tasks, and $\mu \mathrm{C} / \mathrm{OS}$ - II real-time kernel is used for conditioning to realize paralleled execution of many tasks, which improves the reliability and real time of system.

Combined with the hardware structure of the system, according to the priority, the tasks of the system is ordered, signal collection task, data processing and resonance eliminating task, keyboard scanning and liquid display task, network communication task and statistical task.

Task 1: signal sampling task

The task has the highest priority, 0 . The functions include establishing other tasks, censusing initialization of tasks and making A/D conversion.

Task 2: data processing and resonance eliminating task

Real-time requirement of electric operation parameters is low, so the content of the task needs to be implemented by separating from task 1 . The task is to make ferromagnetic resonance analysis on the sampled data, FFT conversion. If there is ferromagnetic resonance failure, it automatically triggers both sides of solid-state relay to instantly break-over PT triangle to break the condition of PT and line resonance and dynamically eliminates ferromagnetic resonance of electric system. If there is single ground connection or over-voltage failure, the buzzer and indicator light sends alarming signal.

Task 3 keyboard scan and liquid display task; The task is to complete human-machine interaction, input control of keyboard and LCD display; Task 4: network communication task; The task processes RS-485 communication incidents. Once there is failure, the system sends the failure information to the remote upper computer; Task 5: statistic task, In system configuration, the constant OS_TASK_ATAT_EN is defined as 1, and the task is established. The task is used to statistic the current utilization ratio of CPU.

Overall structure of system. From the above analysis on the design of hardware and software structure, the system can be divided into three layers, the bottom layer is hardware platform layer including ARM microprocessor system and relating external circuit, the second layer is task layer including circuit driving program and application, and the highest layer is $\mu \mathrm{C} / \mathrm{OS}$ - II operation system level, which is the management core of the system. 


\section{System Protection and Anti-interference Measures}

The working environment of ferromagnetic resonance monitoring system is worse, and electromagnetic interference is serious, so the design takes the following protection and anti-interference measures.

(1) System circuit board is put in metal machine, and the signal lines use shielded wire to eliminate resonance interference and electrostatic induction.

(2) PCB of system circuit board is drawn according to routing rules of high-frequency PCB board to achieve good electromagnetic compatibility effect.

(3) The program design of the system uses redundancy and watchdog technology to prevent from disorder of program or dead cycle.

\section{Conclusions}

The paper analyzes the characteristics of LPC2214 ARM microprocessor for ferromagnetic resonance monitoring system of electric system, and focuses on designing functional requirement of the system, hardware structure and software structure, and system protection and anti-interference measures. The paper makes deep research on hardware resource and LPC2214 chip controlling core ARM, and designs and establishes an embedded system with ferromagnetic resonance detection and analysis of RS-485 data communication interface for actual application. And the paper programs the implementation of hardware and software of the system.

\section{References}

[1] S.Mozafari, M.Sameti. Effect of initial conditions on chaotic ferroresonance in power transformers. IEE Proc., Gener. Transm. Distrib., 1997, 144(5):456-460.

[2] Glenn.W.Swift. An analytical approach to ferroresonance. IEEE Transactions on Power Appratus and Systems, 1969, 88(1):633-637.

[3] J.R.Marti, A.C.Soudack. Ferroresonance in power systems: Fundamental solutions. IEEE PROCEEDINGS-C, 1991, 138(4):345-350.

[4] Frank.S.Young, Ronald.L.Schmid, Petter.I.Fergestad. A laboratory investigation of ferroresonance in cable-connected transformers. IEEE Transactions on Power Appratus and Systems, 1968, 87(5):968-971.

[5] B.A.Mork, D.L.Stuehm. Application of nonlinear dynamics and chaos to ferroresonance in distribution systems. IEEE Transactions on Power Delivery, 1994, 9(2):437-442.

[6] C.Kieny. Application of the bifurcation theory in studying and understanding the global of a ferroresonant electric power circuit. IEEE Transactions on Power Delivery, 1991, 6(2): 112-116.

[7] S.Mozafari, A.C.Soudack. Chaotic ferroresonance in power transformers. IEE Proc., Gener. Transm. Distrib., 1995, 142(3):955-958. 